\title{
Numerical Study of Influence of Fluid Distribution over Heat Storage into Porous Media
}

\author{
Grégoire Bellenot $^{1,2,3}$, Fabrice Bentivoglio ${ }^{1}$, Robin Gautier ${ }^{1}$, Arnaud Bruch ${ }^{1}$, Raphael Couturier ${ }^{1}$, Philippe \\ Marty $^{2}$, Michel Coudrais-Duhamel ${ }^{3}$ \\ ${ }^{1}$ CEA-LITEN \\ 17 rue des martyrs - 38054, Grenoble Cedex 9, France \\ Gregoire.Bellenot@cea.fr; Fabrice.Bentivoglio@cea.fr \\ ${ }^{2}$ Université Grenoble-Alpes - LEGI \\ Domaine Universitaire CS40700 - 38058, Grenoble Cedex 9, France \\ ${ }^{3}$ ALSOLENTECH \\ 15 rue des Genêts Parc St-Exupery - 33700, Mérignac, France
}

\begin{abstract}
This paper presents the first results of an original physical model developed for dual media thermocline heat storage to investigate the influence of fluid distribution over performance. The technology, named "dual-media thermocline heat storage" or "liquidsolid regenerator heat storage" is based on a thermal oil flowing through a double particle-size porous media. The particular dual-media used in a French Alternative Energies and Atomic Energy Commission (CEA) experimental device is considered in this study, composed of sand (3mm diameter) and gravel (30mm diameter). The physical model solves usual mass, momentum and heat equations (NavierStokes) considering multi-dimensional behaviour to take into account flow distribution influence and considering separate thermal behaviour for the fluid phase, the gravel phase and the sand phase. The originality of the model consist in the separation of gravel and sand for the thermal behaviour of the porous media. The first part of the paper is a validation step against experimental data obtained in the CEA experimental device ( $3 \mathrm{~m}$ high storage, $1 \mathrm{~m}$ diameter) that has been specifically modified to study fluid distribution by adding inlet devices to force a non-ideal flow profile,. Then the second part of the paper is dedicated to the numerical study of charges for a 2Daxisymetric cylindrical volumes representing the storage. In one case, a uniform distribution all over one end is done, on the other case a uniform distribution on an axial reduced surface of this end.
\end{abstract}

Keywords: Sensible heat storage, Fluid Distribution, Double grain-size porous media, Computational Fluid Dynamics.

\begin{tabular}{|c|c|c|c|c|c|}
\hline$a$ & Thermal exchange surface density & {$\left[\mathrm{m}^{2} / \mathrm{m}^{3}\right]$} & $t$ & Time & {$[\mathrm{s}]$} \\
$C p$ & Specific heat & {$[\mathrm{J} / \mathrm{kg} \cdot \mathrm{K}]$} & $T$ & Temperature & {$[\mathrm{K}]$} \\
$D$ & Diameter & {$[\mathrm{m}]$} & $u$ & Free flow velocity & {$[\mathrm{m} / \mathrm{s}]$} \\
$E$ & Energy & {$[\mathrm{J}]$} & $V$ & Volume & {$\left[\mathrm{m}^{3}\right]$} \\
$g$ & Gravity & {$\left[\mathrm{m} / \mathrm{s}^{2}\right]$} & $\lambda$ & Thermal conductivity & {$[\mathrm{W} / \mathrm{m} \cdot \mathrm{K}]$} \\
$h$ & Surface thermal exchange coefficient & {$\left[\mathrm{W} / \mathrm{m}^{2} \cdot \mathrm{K}\right]$} & $\Lambda$ & Diffusion term & {$[\mathrm{W} / \mathrm{m} . \mathrm{K}]$} \\
$L$ & Length & {$[\mathrm{m}]$} & $\varepsilon$ & Porosity & \\
$P$ & Pressure & {$[\mathrm{Pa}]$} & $\mu$ & Kinematic viscosity & {$\left[\mathrm{m}^{2} / \mathrm{s}\right]$} \\
$R e$ & Reynold number & & Density & {$\left[\mathrm{kg} / \mathrm{m}^{3}\right]$} \\
$S$ & Surface & {$\left[\mathrm{m}^{2}\right]$} & $\rho$ & & \\
\hline
\end{tabular}




\section{Introduction}

In the global rise of renewable energies, Concentrated Solar Power (CSP) is expected to play a non-negligible part as it permits a constant production of power from an intermittent energy source, thanks to heat storage

A partnership between the French Alternative Energies and Atomic Energy Commission (CEA) and the company ALSOLEN develops a multi-application CSP plant coupling an Organic Ranking Cycle for electricity production, a water desalination unit, a desorption cold-production unit and the production of heat for specific utilities [1]. In such a system the heat storage is a key component, that solves the issues caused by sunshine variability and low predictability, and that provide a high level of flexibility to manage the different production utilities (electricity, heat, cold, fresh water).

A sensible heat storage technology, based on dual-media thermocline, has been chosen. This technology permits to include both hot and cold fluids in the same tank providing two main advantages: reduce expensive material amount and avoid the use of expensive heat exchangers required when the heat transfer fluid is not the same as the storage medium [2]. In this technology, heat storage is made by circulating heat transfer fluid from the top of a porous granular bed made of low-cost high-heat capacity material. Hot fluid from the source (solar field) exchanges heat with solid phase, forming two temperature zones into the storage, separated by a thin horizontal transition layer named thermocline and mainly due to the non-ideal heat exchange. Stored heat recovery is made by reverse circulation of cold fluid, as presented in the Figure 1.

Among the topics investigated in order to improve the concept, influence of fluid distribution is of great interest. It is usually assumed that the distribution of fluid at the inlet of the porous media is a uniform velocity profile leading to the assumption that the flow in the porous media is mono-dimensional (1D). A recent numerical study [3] has shown that a non-uniform inlet profile could lead to better performance for a single particle-size porous media heat storage. This conclusion is of high interest and have to be investigated more in depth, in particular to verify if it can be extended to our concept with double particle-size. In addition, even if the uniform inlet profile proves to be the best option, to obtain such a uniform profile for a large storage such as the one studied by ALSOLEN (diameter $>10 \mathrm{~m}$ ) is quite challenging, and the consequence of a non-uniform distribution over the performance of the storage is also a topic of interest for the concept.

To study the influence of fluid distribution, a physical model has been developed and validated against experimental data coming from a CEA experimental device composed of a $3 \mathrm{~m}$ high - $1 \mathrm{~m}$ diameter vessel filled with a porous media composed of gravel (30mm diameter) and sand ( $3 \mathrm{~mm}$ diameter). The model solves usual mass, momentum and heat equations (Navier-Stokes) considering multi-dimensional behaviour to take into account flow distribution influence and considering separate thermal behaviour for the fluid phase, the gravel phase and the sand phase. The originality of the model consist in the separation of gravel and sand for the thermal behaviour of the porous media. The first part of the paper is a validation step against experimental data obtained with the CEA experimental device that has been specifically modified to study fluid distribution by adding inlet devices designed to produce a non-uniform flow profile over the inlet surface. Then the second part of the paper is dedicated to the numerical study of a charge for a 2D-axisymetric cylindrical volume representing the storage. In one case, a uniform distribution all over the inlet surface is done, on the other case a uniform distribution on a central reduced surface is applied.

\section{Physical system and numerical model \\ 2.1. Development of the numerical model}

The numerical model solves the usual Navier-Stokes equations, applied to the flow of a fluid through a granular porous media composed of two distinct solid phases (sand and gravel), inside a metallic vessel. Several hypothesis are made: homogeneous and isotropic media, no radiation, no thermal exchange between solid phases, one of the solid phases has no thermal gradient inside its particles, unlike the other one.

This leads to solve the equations (1) - (6) using the CFD software FLUENT@. Equations (4) and (5) are additional equations that are added to the default porous model thanks to User-Defined Scalars; they allow to solve temperature equations for both solid phases while considering that the default porous solid of the software only intervene for the resolution of equations (1) and (2). 


$$
\begin{aligned}
& \frac{\partial\left(\varepsilon \cdot \rho_{\text {fluid }}\right)}{\partial t}+\nabla \cdot\left(\varepsilon \cdot \rho_{\text {fluid }} \cdot \boldsymbol{u}\right)=0 \\
& \frac{\partial\left(\varepsilon \cdot \rho_{\text {fluid }} \cdot \boldsymbol{u}\right)}{\partial t}+\nabla \cdot\left(\varepsilon \cdot \rho_{\text {fluid }} \cdot \boldsymbol{u} \cdot \boldsymbol{u}\right)=-\varepsilon \nabla P+\varepsilon \cdot \rho_{f} \cdot g+\left(A \cdot \mu_{\text {fluid }} \cdot \boldsymbol{u}+B \cdot \rho_{\text {fluid }} \cdot|\boldsymbol{u}| \cdot \boldsymbol{u}\right) \\
& \frac{\partial(\text { Cp. E. } \rho . T)_{\text {fluid }}}{\partial t}+\nabla .(\varepsilon . \rho . u . T)_{\text {fluid }} \\
& =\nabla \cdot\left(\Lambda_{\text {fluid }} \cdot \nabla \cdot T_{\text {fluid }}\right)+\sum_{i \in \text { solid phases }}(h \cdot a)_{\text {fluid }, i} \cdot\left(T_{i}-T_{\text {fluid }}\right) \\
& \frac{\partial\left(C p \cdot \frac{V}{V_{\text {tot }}} \cdot \rho \cdot T\right)_{\text {sand }}}{\partial t}=\nabla \cdot\left(\Lambda_{\text {sand }} \cdot \nabla \cdot T_{\text {sand }}\right)+(\text { h.a })_{\text {fluid,sand }} \cdot\left(T_{\text {fluid }}-T_{\text {sand }}\right) \\
& \partial\left(C p \cdot \frac{V}{V_{t o t}} \cdot \rho \cdot T\right) \\
& \partial t \quad \text { gravel }=\nabla \cdot\left(\Lambda_{\text {gravel }} \cdot \nabla \cdot T_{\text {gravel }}\right)+(\text { h.a })_{\text {fluid,gravel }} \cdot\left(T_{\text {fluid }}-T_{\text {gravel }}\right) \\
& \frac{\partial(C p \cdot \rho \cdot T)_{\text {wall }}}{\partial t}=\nabla \cdot\left(\lambda_{\text {wall }} \cdot \nabla \cdot T_{\text {wall }}\right)+(h \cdot a)_{\text {fluid,wall }} \cdot\left(T_{\text {fluid }}-T_{\text {wall }}\right)+h_{\text {loss }}\left(T_{\text {ext }}-T_{\text {wall }}\right)
\end{aligned}
$$

The head loss coefficients are taken from the work of Carman [4] (cited by Erdim [5]), as a good compromise for laminar and turbulent flows, considering that the mean flow in the vessel is laminar and that the flow at the restricted inlet area is turbulent, adapted to a two particle-size granular bed using the approach proposed by Clavier [6].

$$
\begin{gathered}
\frac{\Delta P}{L}=\left(180+2.871 \cdot\left(\frac{R e}{1-\varepsilon}\right)^{0.9}\right) \cdot \frac{(1-\varepsilon)^{2}}{\varepsilon^{3}} \cdot \frac{\mu_{\text {fluid }} \cdot(u)}{d_{\text {sauter }}{ }^{2}} \\
d_{\text {Sauter }}=6 \cdot \frac{V_{\text {solids,total }}}{S_{\text {solids,total }}}
\end{gathered}
$$

Heat transfer coefficients are selected from previous works validated against experimental data with uniform flow (1D) at CEA for this kind of storage. The heat transfer coefficient between fluid and wall "hfluid,wall" is adapted from Dixon et al. as suggested in [7]. The heat transfer coefficient between fluid and gravel "hfluid,gravel" is also from Dixon et al. adapted like [8] and heat transfer coefficient between fluid and sand "hfluid,sand" from Wakao et al. cited in [7]. The free-flow velocity "u" used in these equations is the velocity calculated from volumetric flow rate and total section of the flow.

Diffusion terms " $\Lambda \mathrm{f}$ ", " $\Lambda \mathrm{sa}$ ", " $\Lambda$ gr" are calculated with the method of Cheng and Hsu [9] from the global conductivity of Zehner and Schlünder [10], obtained from conductivity of materials with the method proposed in [11]. These terms are simplified by applying the "tortuosity terms" mentioned is these papers only to the fluid term and by not using the "mixing terms".

\subsection{Description of the experimental device}

The experimental part of this paper is based on the experimental device called "STONE", constructed and operated by CEA LITEN. This device is composed of a cylindrical metallic tank, containing a granular porous media composed of sand ( $3 \mathrm{~mm}$ diameter) and gravel (30mm diameter) and filled with a high temperature thermal oil. This tank is composed of four stacked cylindrical shells, each equipped with a fine grid supporting the porous media. This cylindrical domain is the only numerically computed part of the device. Detailed experimental set-up and physical properties are presented in [12]. 


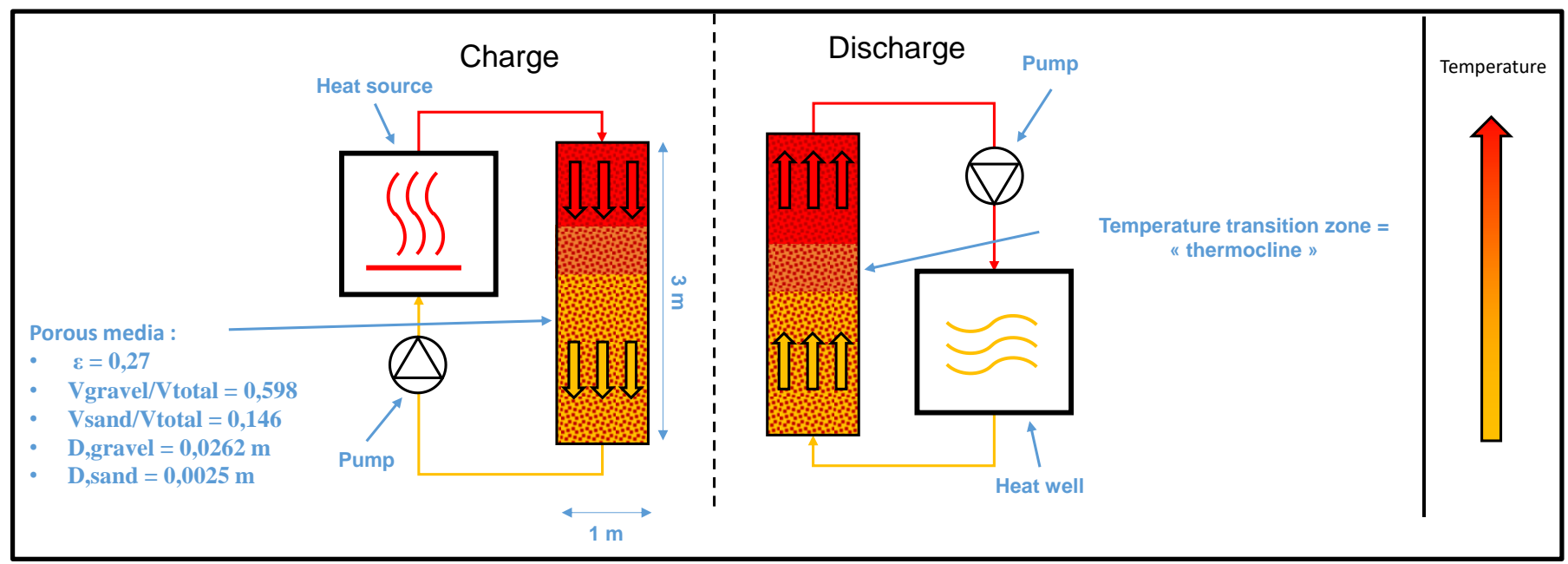

Fig. 1: Schematic of the experimental device "STONE."

The ends of the cylinder are closed by convex ends filled with free volumes of fluid and used for injection and collection of the fluid. The top end is connected to three inlet devices: one large lateral tube and two small ones (one axial, one lateral) that where added after the publication of [12]; the bottom end is only connected to a large lateral tube. These inlet/outlet tubes can be selected to produce different fluid distribution cases. These top and bottom volumes are not calculated in the current version of the model but treated as boundary conditions.

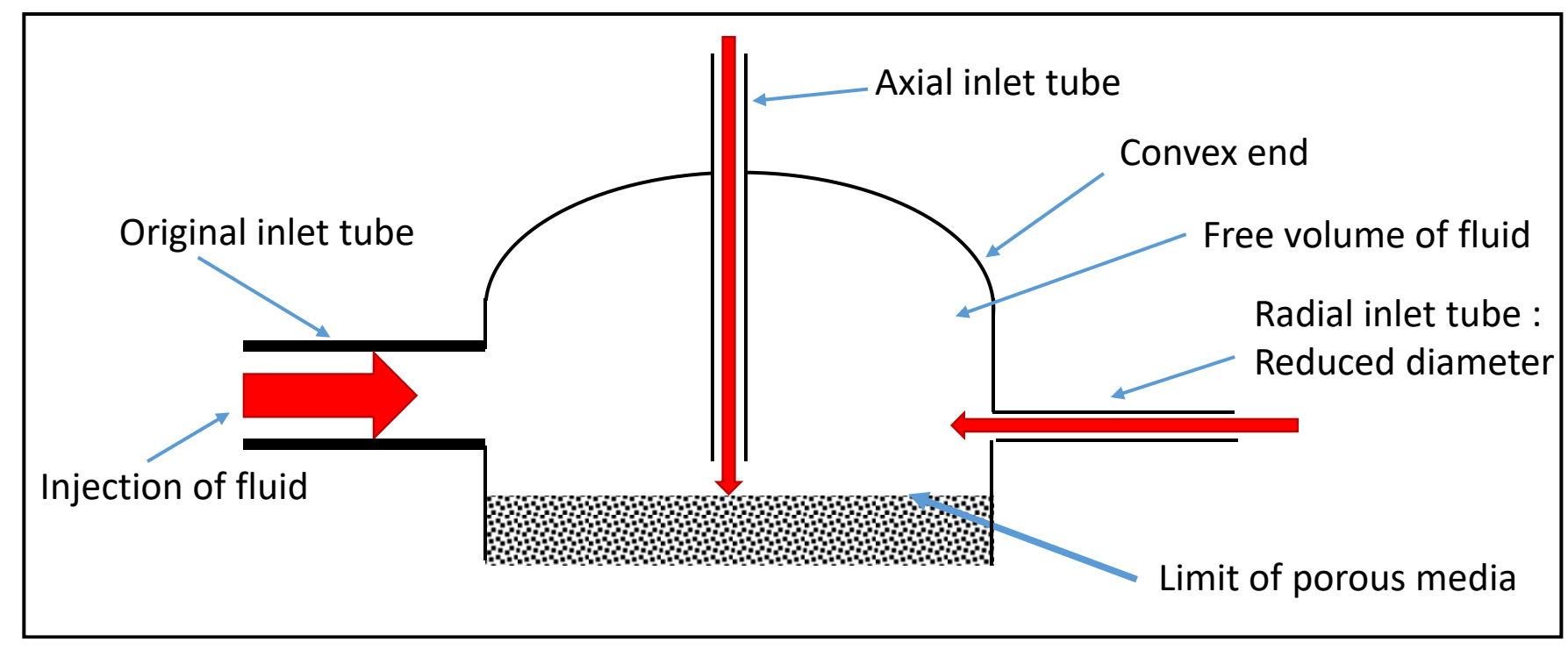

Fig. 2: Schematic of the top convex end of "STONE."

The porous media is instrumented in temperature using thermocouples disposed on several levels along the depth of the reservoir, some measuring fluid temperature, the others the gravel temperature (sand is considered at fluid temperature). 


\section{Validation of the numerical model}

The first part of this study is a validation step against experimental data. Temperatures and mass flow measured throughout experimental runs are used as boundary conditions for the numerical calculations. Temperature measurements measurements are also used to extrapolate temperature profiles which are used as initial conditions.

\subsection{First validation: Complete charge of the storage}

At first, the numerical model is compared to a simple charge of the experimental heat storage with inlet temperature and velocity profile supposed to be uniformly distributed over the top surface of the porous media (use of the large lateral inlet tube). Initialization is done when inlet conditions are stabilized and top convex end can be supposed to be uniformly at maximum charge temperature to avoid any disturbance. This means that a thermal gradient is already installed in the tank at starting time.

Temperature profiles are compared at different times for both fluid and coarse solid phase. Plotted temperatures are arithmetic averages over the section of the tank.

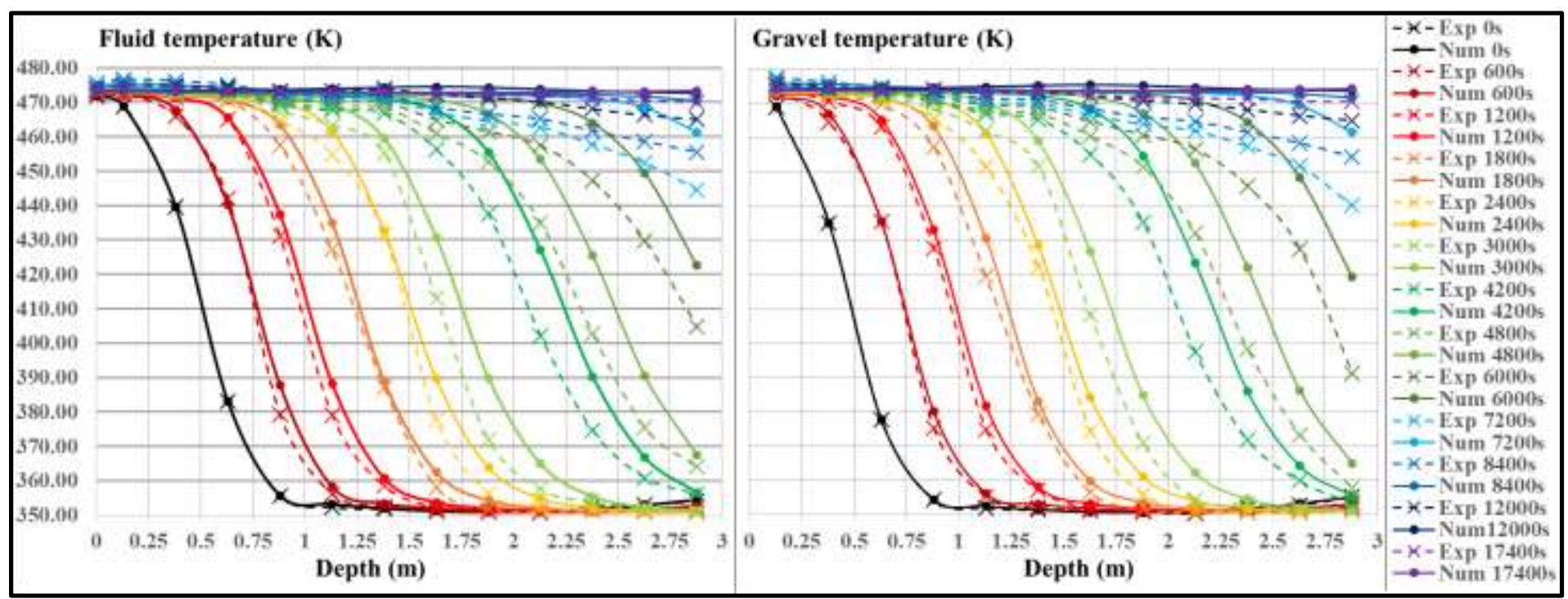

Fig. 3: Comparison of numerical (Num) and experimental (Exp) radial mean temperature profiles for fluid and gravel during first validation case.

One can see that during the first 10th of the test (around $1200-1800$ s), a very good agreement is achieved between model and experiment. On the other side, throughout the rest of the test, experimental temperatures are more and more below numerical ones all along the depth of the tank.

The energy and useful energy contained in the storage is also compared through time. These energies are expressed in equations (9) and (10) and shown in figure 4:

$$
\begin{gathered}
E_{\text {totale }}=\int_{\text {storage }} \rho \cdot\left(\int_{T \min }^{T} C p \cdot d T\right) \cdot d V \\
E_{\text {useful }}=\int_{\text {storage }} \rho \cdot\left(\int_{T \min }^{T} C p \cdot d T\right)_{\text {if } T \geq\left(T_{\max }-10\right)} \cdot d V
\end{gathered}
$$

The useful energy is the energy carried by a temperature above a limit, considered equal to the charge temperature minus $10 \mathrm{~K}$. 


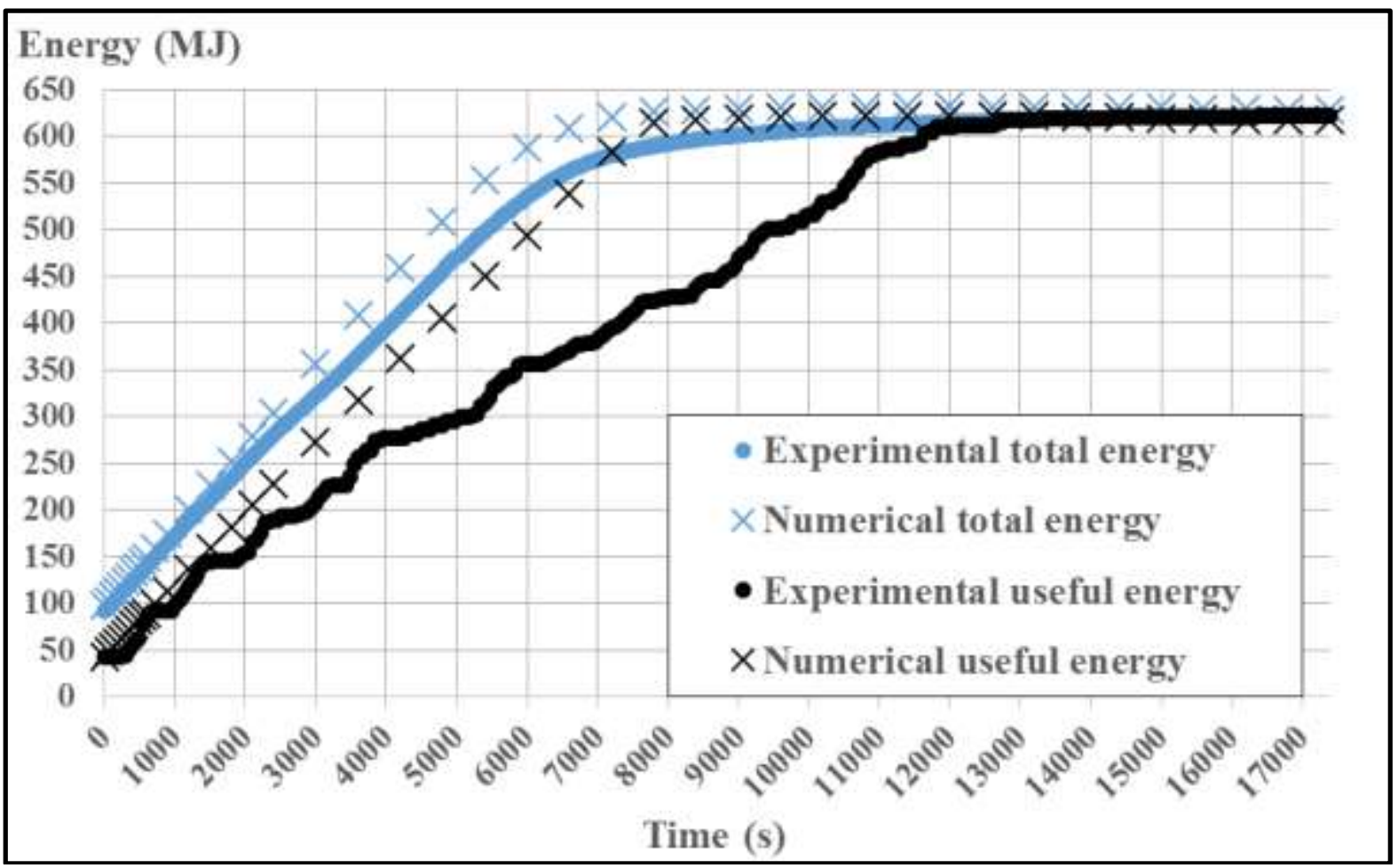

Fig. 4: Comparison of numerical and experimental energy and useful energy during first validation case.

Like temperatures, energy and useful energy are very similar for the first part of the tests and then numerical energies become higher than experimental ones, until they merge at the end. It is noticeable that this difference is higher for useful energy and increase over time before energies reach the maximum value.

Two potential causes are considered to explain these results:

- The fact that differences in energy values start to rise only after a certain time and that the difference is higher for useful energy than for total energy leads to assume the existence of a channeling effect that could participate to the observed behaviour. A part of the fluid would leave the tank with a fraction of its energy, decreasing experimental total energy. Moreover, it would exchange its heat with a larger volume of the porous media along the depth, thickening the thermal gradient and explaining why experimental useful energy becomes significantly lower than numerical one (figure 4) and why numerical temperature curves stay sharper (figure 3 ). The observation of the experimental standard deviation of fluid temperatures along depth of the tank as a function of time seems to corroborate this hypothesis. This also seems confirmed by the observation of solid temperature, level by level, at the times when standard deviation is maximum. It shows that the same zone of the tank is almost always higher in temperature at any level, especially lower ones.

- Another suspected cause for the observed temperature behaviour is the fact that the real experimental porous media is no more uniform along depth. Visual inspection showed that the first quarter of the top shell does not contain any more sand, which can be expected to be the case for each one of them. The rise of porosity and drop of thermal capacity at the top of shells is expected to accelerate temperature fronts for both solid and fluid while it would slow down in bottom parts. This behaviour, occurring at the same time in two to three shells is suspected to participate to the difference between calculations and experimentation but is complex and will necessitate further numerical investigations. 
At the time of redaction of this paper, a second validation case is running with fluid distribution using the axial tube but is not completed enough to be presented here.

\subsection{Conclusions about validation}

Preceding results showed that the developed model is able to simulate the thermal behaviour of the experimental device device STONE. Some discrepancies remain that can be explained by a channelling effect and a non-ideal repartition of sand in the porous media. These possible causes will be investigated in further studies.

The validation case with axial centred jet distribution is under finalization. Another further step in the validation process will be to confront the model to experimental charge/discharge cycles and to several fluid velocities and temperature ranges. Time and grid independence of numerical results will also have to be checked as only the time-step independency of the first validation case have been verified with a ten-times smaller time-step, with differenced in temperatures under $0.05 \%$ and difference in useful energy of $0.025 \%$ of presented results after $1200 \mathrm{~s}$.

\section{Comparison between fluid distribution methods}

After this first step of partial validation, the model can be used to investigate the influence of fluid distribution over heat storage performances. Numerical approach is preferred to experimental setup because it is difficult to produce in the facility two sets of experimental data that are equivalent in inlet mass-flow, temperature profiles and initial conditions because of the effect of top and bottom free fluid volumes.

The calculations are done for a partial charge of the storage. Only the porous media zone is calculated. In both cases, the storage media is initially isotherm at low temperature. Inlet mass-flow and temperature are identical and constant over time. In the first case, fluid inlet is made all over the top surface of the cylindrical porous media (Uniform distribution case); in the second case, it is made through a circular axial surface equal to approximately $0.0676 \%$ of the total surface $(2.6 \%$ of total radius), corresponding to the axial distribution tube (Jet distribution case). In both case, velocity profile is made constant over the inlet surface.

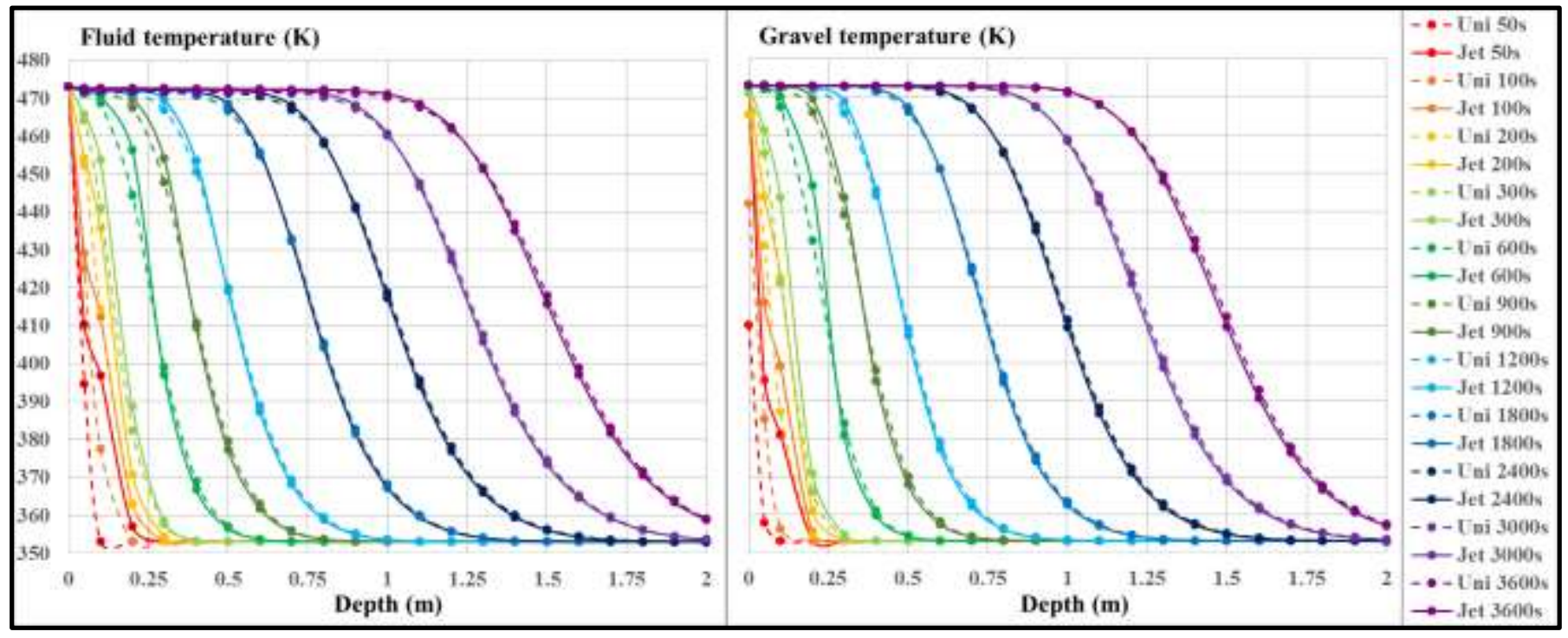

Figure 5: Comparison of numerical radial mean temperature profiles for fluid and gravel during uniform distribution case (Uni) and jet distribution case (Jet). Abscise has been truncated at $2 / 3$ of the depth of the tank.

As can be seen in figure 5, for both fluid and gravel phases, radial mean temperature curves in jet injection case are globally "sharper" in the high temperature gradient zone than in uniform injection case at the beginning of the test but also 
present a flattening. This behaviour disappears with time. This illustrates that the thermocline would be thinner and nonhorizontal in jet injection case at the beginning but becomes larger and recovers its horizontality over time.

This can also be noticed in the curves of energy and useful energy contained in the storage over time (figure 6) during the first half of the test.

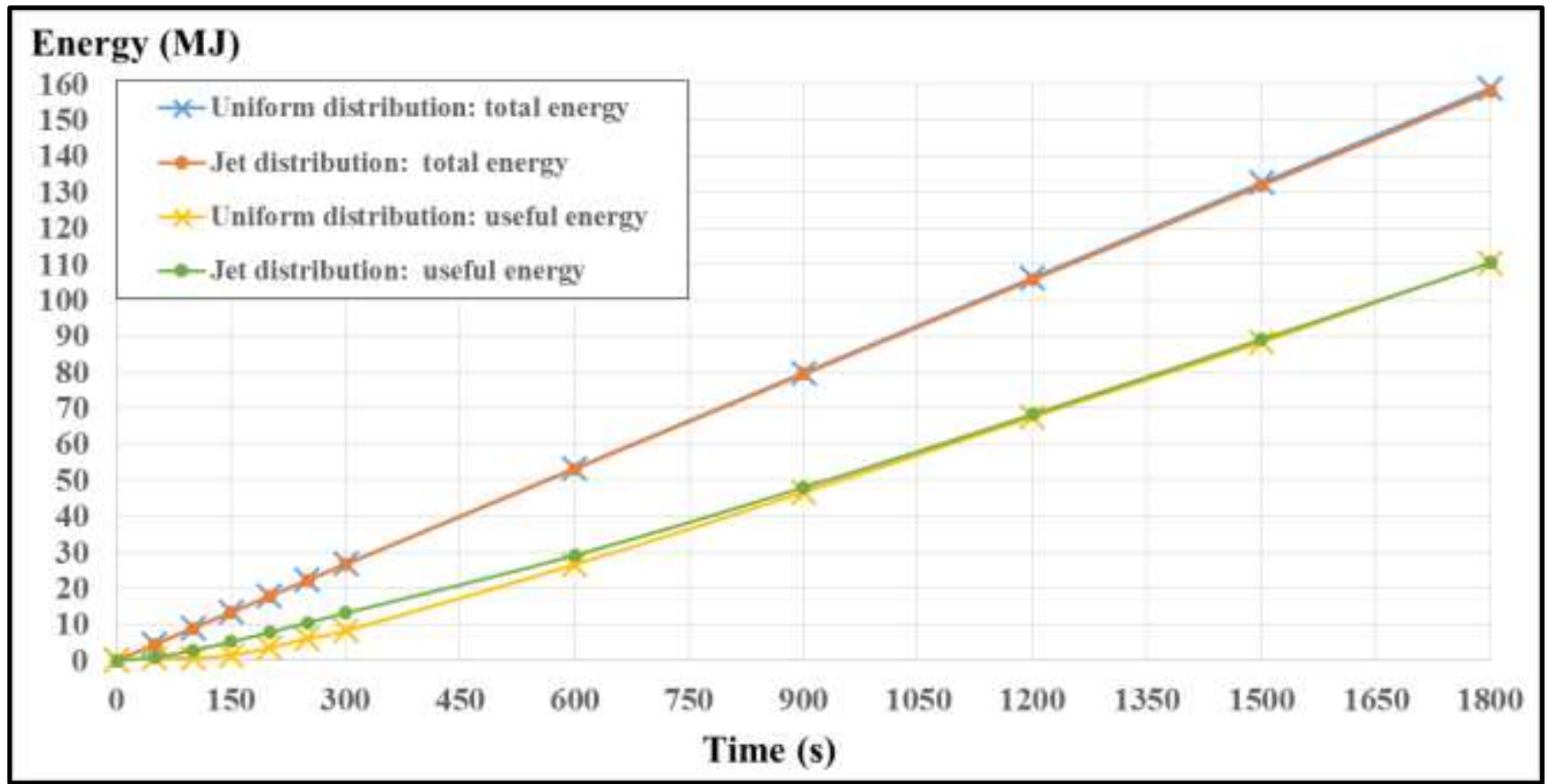

Fig. 6: Comparison of numerical energy and useful energy profiles during uniform distribution case and jet injection case for one half of the duration of the test.

As total energies are almost identical along the test, almost the same energy have indeed been injected in both cases. On the other hand useful energy rises faster for jet distribution than for uniform distribution at the beginning of the test, which means that the same amount of energy is stored but at a higher temperature (better quality). However, useful energy difference start to decrease rapidly until values merge and stay almost equal.

A possible interpretation of the observed behaviour is that two antagonist effects are competing in the jet distribution case.

The fluid jet created by the reduced inlet surface would strongly improves the local exchange between fluid and solid phases, reducing the thermal gradient thickening potential of the flow. On the other hand, this reduced surface inlet distribution would create a temperature gradient over the radius of the media that would enhance mixing by natural convection and thus reduce the quality of the energy. For the first part of the charge, the first effect would be the strongest, explaining the better storage performance of jet injection. After some time, the high gradient temperature zone would be further than the depth where the created fluid jet have been spread all over porous media's radius by head losses so it could not have any more effect on heat exchange. At this point, both cases would become equivalent in term of injection. However, jet injection would have a non-horizontal radial temperature profile that would thicken its thermocline over time, explaining why this distribution loses its advantage over time.

Useful energy in uniform injection case does not seem to become higher than in jet injection case which would mean that the thickening effect is over and that uniform injection would not become better for a longer charge. 


\section{Conclusion}

An original physical model has been developed for two particle-size dual-media thermocline heat storage to investigate the influence of fluid distribution over performance.

This model has been partially validated against experimental data for a charge with uniform inlet velocity profile across across flow section. This validation process is still under progress and will be extended to axial jet velocity profile and different velocities.

A first numerical study concerning the effect of the distribution profile has been carried out. It concludes that a nonperfectly distributed velocity profile is highly better at the beginning of the charge than a perfectly distributed velocity profile, but that this advantage attenuates rapidly and becomes negligible after $25 \%$ of the total charging time.

Two thermal effects are suspected to cause the observed differences in temperature and useful energy profiles between the fluid injection cases. A local improvement of fluid/solid heat exchange, caused by fluid acceleration due to jet velocity profile would reduce thermocline thickening. On the other hand the non-perfectly distributed injection of fluid would create a non-horizontal gradient that would tend to thicken the thermocline by natural convection.

With the unique simulation made it is still not possible to tell if these two effects keep the same respective strength for any mass-flow, temperature, duration, charge/discharge cycling and fluid distribution configurations. Further investigations are expected to determine the operation conditions in which certain distribution solutions are better.

These results may also be applied to the industrial case of ALSOLEN, taking into account its greater complexity.

\section{References}

[1] S. Rodat, A. Bruch, N. Dupassieux, and N. E. Mourchid, "Unique Fresnel Demonstrator Including ORC and Thermocline Direct Thermal Storage: Operating Experience," Energy Procedia, vol. 69, pp. 1667-1675, May 2015.

[2] S. S. Mostafavi Tehrani, Y. Shoraka, K. Nithyanandam, and R. A. Taylor, "Shell-and-tube or packed bed thermal energy storage systems integrated with a concentrated solar power: A techno-economic comparison of sensible and latent heat systems," Appl. Energy, vol. 238, pp. 887-910, Mar. 2019.

[3] L. Wang, Z. Yang, and Y. Duan, "Influence of flow distribution on the thermal performance of dual-media thermocline energy storage systems," Appl. Energy, vol. 142, pp. 283-292, Mar. 2015.

[4] P. C. Carman, "Fluid flow through granular beds," Chem. Eng. Res. Des., vol. 75, pp. S32-S48, 1997.

[5] E. Erdim, Ö. Akgiray, and İ. Demir, "A revisit of pressure drop-flow rate correlations for packed beds of spheres," Powder Technol., vol. 283, pp. 488-504, Oct. 2015.

[6] R. Clavier, N. Chikhi, F. Fichot, and M. Quintard, "Experimental study of single-phase pressure drops in coarse particle beds," Nucl. Eng. Des., vol. 312, pp. 184-190, Feb. 2017.

[7] T. Esence, A. Bruch, S. Molina, B. Stutz, and J.-F. Fourmigué, "A review on experience feedback and numerical modeling of packed-bed thermal energy storage systems," Sol. Energy, vol. 153, pp. 628-654, Sep. 2017.

[8] T. Esence, A. Bruch, J.-F. Fourmigué, and B. Stutz, "A versatile one-dimensional numerical model for packed-bed heat storage systems," Renew. Energy, vol. 133, pp. 190-204, Apr. 2019.

[9] P. Cheng and C. T. Hsu, "3.Heat conduction," in Ingham, D.B. and Pop, I., Transport Phenomena in Porous Media, 1998, pp. 57-76.

[10] P. Zehner and E. U. Schlünder, "Wärmeleitfähigkeit von Schüttungen bei mäßigen Temperaturen Zehner_Schlünder," Chem.Ing. Tech., vol.42,pp.933-941, 1970.

[11] T. Esence, "Etude et modélisation des systèmes de stockage thermique de type régénératif solide/fluide," Grenoble Alpes, CEA/LITEN, 2017.

[12] A. Bruch, S. Molina, T. Esence, J. F. Fourmigué, and R. Couturier, "Experimental investigation of cycling behaviour of pilot-scale thermal oil packed-bed thermal storage system," Renew. Energy, vol. 103, pp. 277-285, Apr. 2017. 